\title{
Particularidades de China para el desarrollo empresarial: reflexión a partir de casos de estudio
}

\author{
Distinctive features of China for business \\ development: findings from case studies
}

\author{
Noemí Martínez Caraballo ${ }^{1}$ \\ Universidad de Zaragoza
}

Recibido el 25 de noviembre de 2009, aceptado el 22 de marzo de 2010

$\mathrm{N}^{\mathrm{o}}$ de clasificación JEL: F20, F23, N80, N85

DOI: $10.5295 / \mathrm{cdg} .100189 \mathrm{~nm}$

Reseña Bibliográfica: MARTÍNEZ CARABALLO, N. (2010): "Particularidades de China para el desarrollo empresarial: reflexión a partir de casos de estudio"; Cuadernos de Gestión, Vol. 10. № 2, pp. 67-80. DOI: $10.5295 / \mathrm{cdg} .100189 \mathrm{~nm}$

\section{Resumen:}

China ha irrumpido como actor principal del escenario global al haber protagonizado, durante las tres últimas décadas, un proceso de transformación económica y social de gran magnitud. Ahora bien, vender productos -sobre todo, del sector de alimentación- de origen occidental en China es, con diferencia, el reto más complicado al que deben enfrentarse las compañias de deciden entrar a operar en este mercado. El principal objetivo de este trabajo es ofrecer una visión panorámica de diversas particularidades de China con el fin de conocer su potencial para el desarrollo de negocios occidentales. Para ello, se presentan diversos casos de estudio de empresas que han triunfado al introducirse en el mercado chino y se plantean también ejemplos de decisiones erróneas, pretendiendo servir de reflexión y planteando recomendaciones para los gestores de empresas.

Palabras clave:

China, Gestión multicultural, Inversiones en el extranjero, Penetración en el mercado.

\begin{abstract}
:
China has emerged as a major player on the global scenario, having lead, during the last three decades, a great magnitude process of economic and social transformation. Nevertheless, selling Western produce -mainly, food products-in China is, the most complicated challenge which companies that decide to operate in this market should face. This paper aims to offer a panoramic vision of several China particularities with the purpose of reviewing its potential for Western business development. Hence, a number of cases studies of companies that have succeeded to enter the Chinese market and different examples of wrong decisions or failure cases are presented, trying to provide an analytical evaluation and managerial guidelines.
\end{abstract}

Keywords:

China, Crosscultural Management, Foreign Investments, Market Penetration.

\footnotetext{
${ }^{1}$ Departamento de Economía y Dirección de Empresas, Universidad de Zaragoza, C/ Gran Vía, 2 - 50005 Zaragoza (España). E-mail: noemar@unizar.es. La autora desea agradecer el trabajo realizado por la editora de la revista, Belén Vallejo Alonso, y las sugerencias vertidas por los dos revisores anónimos que han contribuido a mejorar la versión inicial del documento.
} 


\section{INTRODUCCIÓN}

China ha experimentado, durante las tres últimas décadas, una de las transformaciones económicas más rápidas y espectaculares de la historia moderna, con tasas de crecimiento superiores al diez por ciento anual (Quer et al., 2007b). Dicho proceso de transformación está provocando que China se encuentre entre los países preferidos para los negocios internacionales (KPMG, 2008; PriceWaterhouseCoopers, 2008, 2009; Ernst \& Young, 2008, 2009). No en vano, resulta ser la tercera potencia económica del planeta y podría decirse que el centro de gravedad de la economía mundial se está desplazando inexorablemente hacia dicho país (Sánchez-Monasterio, 2009a; Quer et al., 2009).

Sus ventajas en coste y ser el país más poblado del mundo con alrededor de mil trescientos millones de habitantes -trescientos millones de los cuales pertenecen a la clase social media-alta- han permitido bautizarlo como "el mercado potencial más importante del mundo". Por ese motivo, desde principios de la década de los ochenta, España ha realizado un esfuerzo notable y sostenido por desarrollar su presencia en China. Ahora bien, dicha presencia de las empresas españolas en China es aún baja si se tiene en cuenta tanto el atractivo del destino como el potencial del tejido empresarial español (Bustelo, 2006).

Bueno et al. (2006) señalan que cuatrocientas compañías españolas están presentes en este país asiático, pertenecientes a los siguientes sectores: alimentación (Nutrexpa, Panrico, Chupa Chups, Miguel Torres, etc.); bienes de consumo (Lladró, Adolfo Domínguez, Roca, Indo, Mango, Zara, Fagor, Fermax, etc.); transporte y material de transporte (Alsa ${ }^{1}$, Antolín, Indra, Abengoa, Ficosa, etc.), energía eólica (Gamesa, Acciona, etc.) ingeniería (Técnicas Reunidas, Gestam, etc.), construcción (Sacyr Vallehermoso, etc.); bienes de equipo (Mondragón, etc.) y el sector bancario (BBVA, Santander, Bancaja, Caja Madrid, etc.).

La creciente importancia de China en el escenario global no ha pasado desapercibida por parte del mundo académico. Así, existen diversos trabajos dedicados a analizar los determinantes del éxito/fracaso de entrada en países emergentes como China o India (véase, por ejemplo, el trabajo de Johnson y Tellis, 2008). Ahora bien, la mayor parte de ellos se han centrado en la dirección de empresas en China, en particular de las joint ventures establecidas en el país (e.g., Quer et al., 2007a y 2007b).

Además, merece la pena destacar que este tema ha sido -y continúa siendo- abordado desde la interdisciplinariedad en el sentido que se ha intentado arrojar luz tanto desde el área de conocimiento de comercialización e investigación de mercados como desde el área de organización de empresas, siendo vasta la literatura existente sobre este tema desde la dirección estratégica y la internacionalización de la empresa (e.g., Root y Ahmed, 1979; Dunning, 1988; Zhao et al., 2004).

Puesto que China presenta una serie de peculiaridades que influyen en el éxito logrado en la gestión empresarial, este trabajo analiza el atractivo que muestra dicho destino para la inversión extranjera española y las particularidades de este mercado para la consolidación de negocios en territorio asiático. Con la pretensión de mostrar evidencias sobre los elementos que influyen en una gestión empresarial exitosa, se presentan diversos ejemplos

\footnotetext{
${ }^{1}$ Véase el trabajo de Cosmen (2002), contenido en el número monográfico de Información Comercial Española: Revista de Economía titulado China en el siglo XXI.
} 
que ponen de manifiesto la importancia de la adaptación cultural en el desarrollo del marketing internacional. La experiencia de empresas que no han logrado el éxito esperado al operar en China y conocer las vías de solución que han encontrado puede aportar algunas lecciones sobre lo que no se debe hacer. Finalmente, se vislumbran los retos de futuro que plantea el despertar de este país.

En resumen, la principal aportación del presente trabajo proviene: (1) de la recopilación de información de fuentes secundarias diversas dirigidas a plasmar la evidencia empírica obtenida y (2) del valor añadido ofrecido por los ejemplos que tratan de ejemplificar aciertos y errores cometidos por empresas occidentales que han tratado de realizar su incursión en China tratando de presentar pautas de acción útiles para los gestores de empresas españolas que tienen planteado empezar a operar en el mercado chino tras haber observado el atractivo que tiene China como mercado.

\section{ATRACTIVO DE CHINA COMO MERCADO}

Durante las primeras décadas del siglo XXI, la región de Asia y el Pacífico está teniendo y va a tener un atractivo importante para las empresas internacionales. Se proyecta que la región llegue a ser una potencia tecnológica, política y económica con el incremento simultáneo de la demanda de los consumidores (Czinkota y Ronkainen, 2002).

Según la encuesta realizada anualmente por la consultora A.T. Kearney, China lidera en el año 2010, y desde el año 2003, el ranking del Foreign Direct Investment (FDI) Confidence Index. Ello es debido a que China goza de una serie de ventajas a medio y largo plazo: su elevada integración en la economía mundial, sus buenas infraestructuras físicas, un modelo de desarrollo que genera muchos puestos de trabajo y una relativa estabilidad macroeconómica (Bustelo, 2008). Dichas ventajas proceden, en gran medida, del modelo chino de desarrollo, esto es, del denominado Consenso de Pekín, que tiene como componentes clave el "capitalismo de Estado", gradualismo, apertura al exterior, autoritarismo político y capacidad de innovación y flexibilidad (Fanjul, 2009).

Tal y como ilustra la Tabla 1, las tasas de crecimiento del Producto Interior Bruto (PIB) en China durante los últimos años se sitúan en torno al diez por ciento, brindando importantes oportunidades a las empresas que decidan establecerse en este país.

Tabla 1

Evolución del Producto Interno Bruto - Tasa de Crecimiento Real para el período 2004-2009*

\begin{tabular}{|c|c|c|c|c|c|c|}
\cline { 2 - 7 } \multicolumn{1}{c|}{} & $\mathbf{2 0 0 4}$ & $\mathbf{2 0 0 5}$ & $\mathbf{2 0 0 6}$ & $\mathbf{2 0 0 7}$ & $\mathbf{2 0 0 8}$ & $\mathbf{2 0 0 9}$ \\
\hline China & $9,1 \%$ & $9,1 \%$ & $10,2 \%$ & $10,7 \%$ & $11,9 \%$ & $9 \%$ \\
\hline
\end{tabular}

* Esta variable recoge el crecimiento anual del PIB ajustado por la inflación, expresado como un porcentaje. Fuente: The World Factbook (2009)

Veamos ahora cuál ha sido el papel de la inversión en el crecimiento económico. El crecimiento experimentado durante estos últimos años se ha sustentado en la inversión. 
En la Tabla 2 se muestran los flujos de inversión española en China y de inversión china en España.

Tabla 2

Flujos de inversión extranjera directa para el período 2004-2009

(Datos en miles de euros)

\begin{tabular}{|c|c|c|c|c|c|c|}
\hline & $\mathbf{2 0 0 4}$ & $\mathbf{2 0 0 5}$ & $\mathbf{2 0 0 6}$ & $\mathbf{2 0 0 7}$ & $\mathbf{2 0 0 8}$ & $\begin{array}{c}\mathbf{2 0 0 9} \\
\text { (enero-septiembre) }\end{array}$ \\
\hline $\begin{array}{c}\text { Inversión } \\
\text { Española en China }\end{array}$ & $69.281,94$ & $63.971,76$ & $149.745,42$ & $620.039,48$ & $96.146,60$ & $64.397,83$ \\
\hline $\begin{array}{c}\text { Inversión China } \\
\text { en España }\end{array}$ & 971,41 & 609,63 & $1.320,90$ & $2.154,54$ & $1.047,29$ & $1.876,78$ \\
\hline
\end{tabular}

Fuente: DATAINVEX

La Inversión Exterior Directa (IED) de España en China alcanzó el volumen máximo en el año 2007, si bien, actualmente se ha visto disminuido muy notablemente debido a la crisis. Ahora bien, la presencia española en China es aún inferior a la que cabría esperar, por motivos tales como el idioma, las diferencias culturales o la propia idiosincrasia del país (La Caixa, 2006; Quer et al., 2009).

El atractivo de China como mercado objetivo reside en que se trata de un vasto mercado con una clase media-alta ("Zhong he Gao Shou Ru") de trescientos millones de personas que busca, a través de la compra de marcas occidentales, satisfacer sus necesidades de reconocimiento social, prestigio y estatus ${ }^{2}$. La clave, en palabras de Sánchez-Monasterio (2009a), se encuentra en optimizar el posicionamiento de las marcas y productos occidentales en la mente del consumidor chino como respuesta a sus necesidades. Basándose en datos demográficos, se estima que en la próxima década el número de viviendas con habitantes de la clase social media-alta y edad entre 30 a 60 años se incremente en un $128 \%$, lo cual supone un mercado potencial que no resulta en absoluto despreciable. La previsión es que, en el año 2020, exista en China una clase media-alta de seiscientos millones de personas (Sánchez-Monasterio, 2009b).

Asimismo, el sector de venta minorista se encuentra más desarrollado en las áreas densamente pobladas de las ciudades, donde también se registra la mayor concentración de jóvenes profesionales. Estas áreas son el principal foco de venta de los productos occidentales. La urbanización, los cambios de estilo, el desarrollo de nuevos canales de comercialización y comunicación, también diferencian el patrón de consumo. Ahora bien, aunque China se esté urbanizando rápidamente, continúa siendo un país de extremos y las fórmulas de negocio que resultan válidas en otros países pueden no serlo si se decide exportar la fórmula a China y replicarla sin haber tenido previamente en cuenta una serie de particularidades.

\footnotetext{
${ }^{2}$ Los empresarios chinos y la "beautiful people" china -poseedores del nivel máximo de renta- se han convertido en un target prioritario de las marcas de lujo occidentales. China es el tercer consumidor, a nivel mundial, de artículos de lujo, después de Estados Unidos y Japón (Sánchez-Monasterio, 2009a).
} 


\section{CLAVES PARA UNA GESTIÓN EMPRESARIAL EXITOSA EN CHINA}

\subsection{Elementos culturales que influyen en la gestión empresarial y su éxito}

Las empresas necesitan adaptarse a los mercados donde actúan. Harris y Moran (2001) plantean diez conceptos clave necesarios para el liderazgo global, es decir, para empresas que trabajan en entornos multiculturales. Dichos conceptos son: liderazgo global, comunicación intercultural, sensibilidad cultural, aculturación (definida como el ajuste y adaptación efectiva a una cultura específica, ya sea una subcultura dentro del propio país, o bien, en el extranjero), influencias culturales en la gestión, desempeño intercultural efectivo, cambio en los negocios internacionales, sinergia cultural, cultura de trabajo y cultura global. La comprensión y utilización de estos conceptos es crítica para el desempeño exitoso a escala internacional.

Según Grande (2004), los motivos que inducen a la adaptación cultural son diversos: entorno físico, entorno legal, entorno cultural, percepciones, lengua, gustos, valores éticos y religiosos, etnocentrismo, símbolos y formas de comunicación.

A continuación, se destacan los principales elementos culturales que deberían tener en cuenta las empresas que deciden comercializar y vender sus productos en China:

- Entorno físico. China, debido a su gran tamaño, presenta multitud de contrastes y podemos hablar de un conjunto de diferentes mercados con características propias que atraviesan un proceso de transformación paralelo al que experimenta la sociedad. Así, por ejemplo, el Oeste de China, sin una infraestructura desarrollada, desértico y con menor densidad poblacional, está menos expuesto a los productos occidentales.

- Entorno legal. La República Popular China es una república socialista gobernada por el Partido Comunista de China. En la década de 1970 se introdujeron una serie de reformas dirigidas al mercado que, entre otras, culminaron con la aprobación de la Sino-Foreign Equity Joint Venture Law en 1979. Dicha ley estableció el marco jurídico para regular la inversión extranjera. Durante los años siguientes, la forma más común para una empresa extranjera de operar en China era contar con un socio local que cuente con relaciones y contactos con las autoridades. Hoy en día, y de manera creciente, la inversión extranjera directa entra en China en forma de empresas cien por cien extranjeras.

- Entorno cultural. La gran extensión territorial de la República Popular China tiene como consecuencia una gran diversidad cultural. En el terreno de los negocios, cabe destacar la importancia de forjar relaciones o redes interpersonales, lo que se conoce por guanxi (Adams, 2007; Quer et al., 2009). "Guan" significa "puerta” y “xi” significa "lazos”. Semánticamente se puede definir como una puerta o una conexión entre dos personas creada por una mutua obligación entre ellas. Para los empresarios extranjeros que trabajan en China, construir elementos de confianza es aún más urgente que para los gerentes locales. Esta red de relaciones resulta clave para establecer alianzas empresariales y para los contactos con la administración, los clientes, proveedores y empleados (Hernández-Nieto, 2006). Por ello, es necesario entender la importancia del guanxi, renqing -o reciprocidad- y xinyong - o confianza- a la hora de realizar transacciones comerciales en China (para una revisión en profundidad de estos conceptos, véase el trabajo de Ordoñez de Pablos, 2004). Conocer los protocolos y forjar relaciones confiables son pasos que no se pueden obviar en el proceso de desarrollo del guanxi en una compañía extranjera que quiera incursionar en China. 
- Los símbolos varían según las culturas. En China, por ejemplo, los animales se perciben como portadores de felicidad, el color gris se reserva para los objetos baratos y el número 8 (八) es percibido como el número de la fortuna. La pronunciación fonética del número "8" es "bā", exactamente la misma pronunciación que la palabra "Progreso" y "Fortuna". Por esta razón es frecuente ver, en establecimientos comerciales, precios de venta al público de sus productos en los que predomina dicho número. La inauguración de los Juegos Olímpicos de Beijing fue el 8 de agosto de 2008 a las 8:08 de la tarde. Comprar un número de teléfono móvil de China Unicom en el que predomina el número 8 cuesta hasta 3 veces más que cualquier otro número de teléfono móvil.

- Las formas de comunicación y negociación varían por culturas. Existen una serie de elementos culturales que resultan relevantes para las empresas extranjeras -en concreto, españolas- que deciden establecerse en China. Así, siguiendo a Bhasin (2007) y Quer et al. (2009), se deben tener en consideración los siguientes aspectos:

- Relaciones jerárquicas y estilo de comunicación muy formal.

- No hay sitio para el individualismo.

- La confianza y las relaciones personales (guanxi) son vitales.

- Tienden a adoptar una visión a largo plazo.

- Mirar fijamente les incomoda.

- Tienen menos experiencia sobre el sistema legal moderno.

\subsection{Diseño de un plan de marketing diferenciado}

Además de adaptarse a las diferencias culturales, el éxito de las empresas extranjeras en China pasa por tener en cuenta otras consideraciones. Por ejemplo, dirigirse a los consumidores de clase media, forjar relaciones locales, aprovechar las redes globales y trazar una vía flexible de salida, pueden ser recomendaciones útiles para establecerse con éxito en este mercado (Adams, 2007). Además, la competencia, tanto de multinacionales extranjeras como de las propias compañías chinas, es muy elevada (Quer et al., 2009).

Ahora bien, es necesario que se tengan en consideración una serie de conceptos clave a incluir en el plan de marketing para posicionarse con éxito en este mercado. Lo que se convierte en un desafío complejo es la necesidad de posicionar el producto de tal modo que sean aceptados por el mayor número de consumidores. Así, por ejemplo, conviene prestar atención a las decisiones sobre precio. Cada consumidor chino tiene, para cada producto, un precio máximo que está dispuesto a pagar y un precio mínimo del que si se baja se genera desconfianza sobre la calidad real del producto. Además, existen diferencias considerables entre el precio de venta para el público en China y el precio en Europa. Por ejemplo, el precio en Europa de una Coca Cola es seis veces superior al precio en China (Sánchez-Monasterio, 2009b).

No podemos olvidar que, en China, "Guke shi Shàng Di”, es decir, el cliente es Dios (Sánchez-Monasterio y Casaburi, 2008a). Los clientes se convierten en el eje de cualquier estrategia de marketing. Cabe destacar que el merchandising resulta ser, en China, una herramienta muy eficaz para incentivar la compra del producto, algo que no suele ocurrir en Europa (Sánchez-Monasterio, 2009c). El enorme potencial del merchandising beneficia en gran medida a aquellos productos occidentales que, por razones culturales, no forman parte del día a día del consumo en China (Sánchez-Monasterio y Casaburi, 2008b). 
Analizaremos, ahora, la política de comunicación adecuada para el mercado de China. A este respecto, aquellas empresas interesadas en iniciar su actividad en este país, deben tratar de ofrecer respuesta a una serie de cuestiones como, por ejemplo, ¿debemos traducir nuestra marca occidental al chino? Se muestran, en el presente trabajo, diferentes ejemplos que ponen en relieve la necesaria traducción de las marcas de origen occidental al chino.

Las diferencias lingüísticas resultan ser de vital importancia en el sudeste asiático para la elección de nombres de marca. Mientras que en Occidente un buen nombre sería aquél que fuese corto, distintivo, fácil de recordar e indicativo de las funciones del producto; en Asia, hay que tener consideraciones adicionales que complican el proceso de elección del nombre de marca. Así, un nombre de marca tendrá un impacto significativo en la actitud hacia la marca y, por ende, triunfará en China si éste tiene buena presentación y está bien escrito. En general, que el nombre de la marca tenga connotaciones positivas funciona en la mayoría de los casos. La tarea de encontrar un nombre chino adecuado para una marca occidental muchas veces se delega en algún socio local. Sin embargo, con frecuencia, las empresas simplifican el proceso y pierden la oportunidad de cargar a la nueva marca con la visión y valores que ya tiene como hicieran Carrefour, L'Oréal, Volkswagen, Coca-Cola y Cola-Cao, entre otros. En la Tabla 3 se resume la evolución en nombre de marca que han experimentado dichas empresas para penetrar con éxito en el territorio chino.

Tabla 3

Adaptación de las marcas occidentales al chino

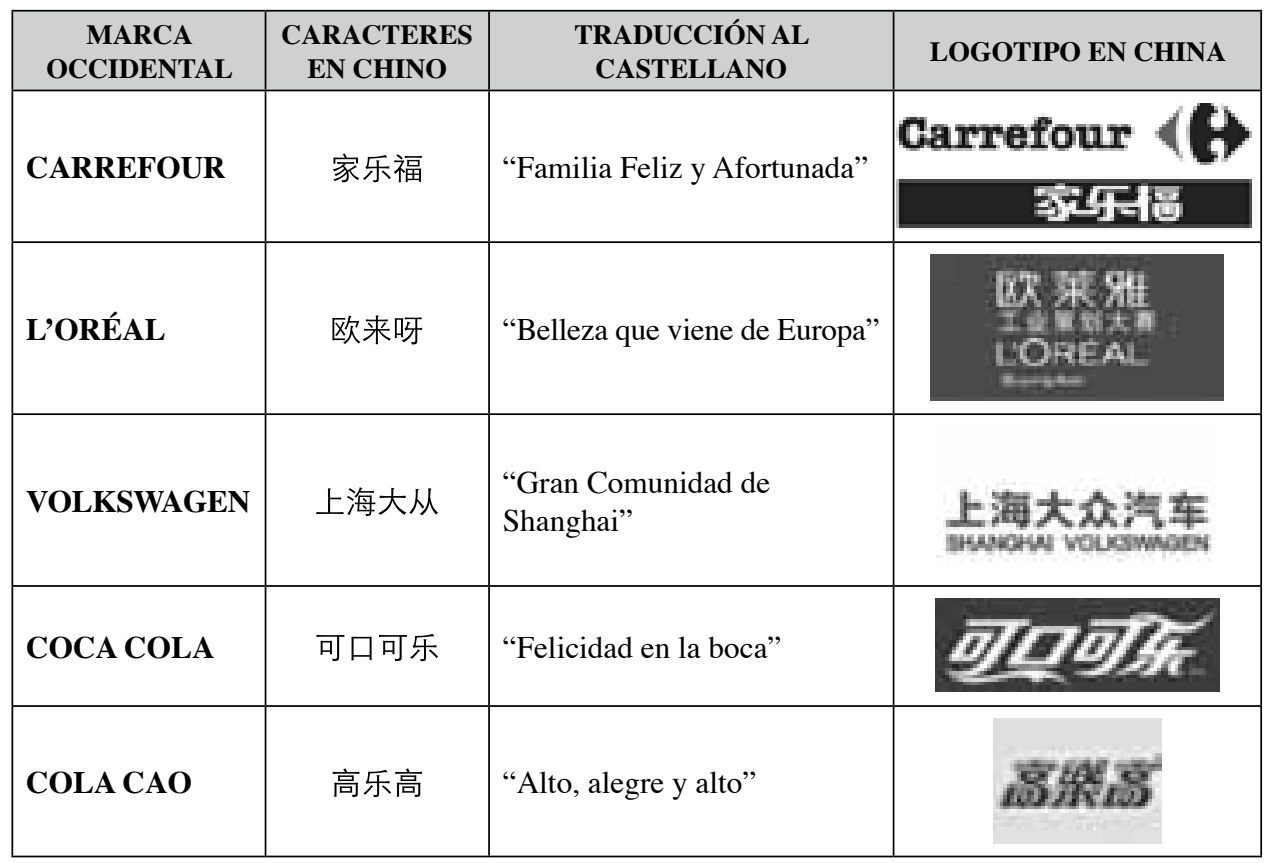

Fuente: Elaboración propia. 
Otro ejemplo sería “Yi Jia Jia Ju” (宜家家居) que, traducido al castellano significa: “Lo apropiado para la casa”. La marca occidental es Ikea.

\section{EVIDENCIA EMPÍRICA A PARTIR DE CASOS DE ESTUDIO}

En este epígrafe se presentan cuatro casos de estudio para mostrar evidencia acerca de cómo la consideración de las particularidades del mercado chino suponen una ventaja - $\mathrm{O}$ desventaja- para las empresas de cara a entrar en este mercado y competir con las empresas locales y extranjeras asentadas en el país.

En particular, el ejemplo de Coca-Cola muestra cómo la elección de nombre debe ser considerada una tarea prioritaria en el plan de comunicación de las empresas que deciden exportar su fórmula de negocio. El caso de Cola Cao corrobora la importancia de la elección de un buen nombre para el producto y aporta evidencias sobre cómo debe actuar una empresa para adaptar sus productos al mercado local, tras haber detectado que dicho producto no satisface los gustos de la población china. Nutrexpa con su Cola Cao logró adaptar el producto a las necesidades y hábitos de consumo locales. Starbucks Corporation demuestra que la falta de consideración de los valores éticos y religiosos de los consumidores puede tener un impacto negativo en la imagen de la empresa. El último de los casos de estudio, que versa sobre la red social Facebook, muestra cómo el fenómeno de la censura y las desventajas que tienen las empresas occidentales al tratar de competir con compañías locales ya asentadas en el gigante asiático.

\subsection{Coca-Cola en China}

Cuando, en 1928, Coca-Cola entró por primera vez en el mercado chino, no tenían una representación oficial de su nombre en chino mandarín. Mientras la empresa buscaba una combinación satisfactoria de símbolos para representar su nombre, los comerciantes chinos crearon signos combinando caracteres. Coca-Cola fue inicialmente presentada en China como "Ke-kou-ke-la" y la compañía no descubrió hasta haber impreso miles de anuncios que dicha representación significaba "muerde el renacuajo de cera". Entonces, Coca-Cola encontró uno que sonaba parecido: “Kekou-kele”. "Kekou”, 可口, significa sabroso, delicioso de comer o beber y "kele”, 可乐, significa felicidad. Podríamos traducirlo por "felicidad en la boca”. Finalmente, se usaron dichos caracteres: 可口可乐, resultando ser ésta la forma en que Coca-Cola registró su marca en China en 1928.

Ya en 1988, Coca-Cola presumía de estar utilizando una estrategia de marketing internacional basada en el lema "Piensa globalmente, actúa localmente", en palabras del vicepresidente de Coca-Cola: Ira C. Herbert ${ }^{3}$. En concreto, manifestaba estar llevando a cabo una estrategia parecida pero no igual en la comercialización de sus productos.

\footnotetext{
${ }^{3}$ Véase la entrevista llevada a cabo a Ira C. Herbert, objeto de publicación en: "How Coke Markets to the World: An Interview with Marketing Executive Ira C. Herbert”, Journal of Business Strategy, 1988, 9 (5), pp. 4-7.
} 


\subsection{Cola Cao en China}

Fabricar para comercializar y vender en China es bastante complicado, especialmente en el sector alimentario y de productos de gran consumo. La razón es ese mítico mercado de mil trescientos millones de consumidores. Aunque este país presenta rasgos comunes en todo su territorio, es fundamental que la estrategia empresarial de las empresas españolas aborde la presencia en China de forma segmentada (Hernández-Nieto, 2006).

Un buen ejemplo, en este sentido, es Nutrexpa que aterrizó en septiembre de 1990 en Tianjin (China) con su producto Cola Cao y ahora, veinte años después, ostenta el liderazgo en el mercado de polvo de cacao, con una cuota del sesenta por ciento que deja atrás a sus competidores: Nestlé, Cadbury y Unilever.

Una facturación de más de treinta millones de euros en China, que representa el $10 \%$ de la del grupo, pone de manifiesto el éxito logrado por este grupo empresarial. Este buen resultado se descompone en lo siguiente: un producto que llega a todas las provincias chinas, con distribuidores en doscientas ciudades y presencia en más de veinte mil tiendas.

Sin embargo, lograr este resultado no ha sido tarea fácil. Cuando Nutrexpa empezó a vender en China, la empresa llevaba dos años batallando con el registro de su marca. De entrada, "Cola Cao" no funcionaba fonéticamente y hubo que rebautizarlo como "Ko Le Kou". Ese nombre, que en chino significaba "proporciona alegría en la boca", colisionaba con "Koke Koule", el seudónimo que usaba en China "Coca Cola" y hubo que rebautizarlo como “Gao Le Gao" (高乐高), que significa, “Alto, Alegre, Alto”. En casos como éste, los expertos aconsejan utilizar una estrategia a largo plazo así como registrar el nombre en castellano y en chino. Es importante registrar el nombre en castellano y en chino, con la grafía y la fonética de aquel país. Y por último conviene fijar cláusulas de arbitraje que se diriman siempre en el mismo tribunal.

Dos grandes retos a los que tuvo que enfrentarse Nutrexpa fueron que (a) en China no se consumía leche y la poca que consumían era en polvo y (b) que el consumidor chino es poco fiel a las marcas y bastante impulsivo. Consecuentemente, el Cola Cao ha de ser soluble en leche en polvo, que era la usada por la mayoría de chinos, así que la textura y tamizado del producto han de permitir que éste se disuelva sin problemas. Es más, se puede observar la poca "adaptación" al binomio leche-Cola Cao en que tienen que poner las instrucciones de uso en el envase. Por eso, Cola Cao decidió lanzar, en China, cinco sabores diferentes: fresa, vainilla, plátano, naranja y melocotón además del tradicional Cola Cao de chocolate, lo cual ha derivado en que los potenciadores de sabor de la leche ${ }^{4}$ supongan, aproximadamente, un tercio de sus ventas.

Resumiendo, podría decirse que gran parte del éxito de la empresa reside en haber sabido aprovechar el momento en el que el Gobierno chino realizó una campaña institucional para fomentar el consumo de lácteos y en haber sabido adaptar su oferta de productos a las necesidades de este mercado.

\footnotetext{
${ }^{4}$ Se comercializan muchos sabores de Nesquik y de Cola Cao fuera de España y sólo de chocolate en territorio español.
} 


\subsection{Starbucks Corporation en China}

El caso de Starbucks es un ejemplo de cómo una compañía occidental puede triunfar superando las barreras culturales, especialmente en países del Este u Oriente. Starbucks Corporation o “xīngbākè" (星巴克) en chino es una empresa dedicada a la compra, tostado y comercialización de café fundada en Seattle en 1971. En 1999, Starbucks abrió su primer establecimiento en China, en el China World Trade Center de Pekin.

El café ha sido tradicionalmente una bebida muy poco popular entre la población china, la cual tiene una amplia cultura como bebedores de té. Sin embargo, este hecho no es tanto por los gustos de la población china, sino por la falta de hábito o costumbre, ya que la mayoría nunca ha probado un café de calidad.

La estrategia que adoptó Starbucks fue intentar atraer a sus potenciales clientes -una nueva generación con una creciente capacidad adquisitiva y apetito por marcas exclusivasaportando un lugar informal de reunión y ofreciendo no ya la experiencia de consumir café, sino la experiencia de estar en un establecimiento Starbucks.

Muchos de sus establecimientos en China son de primera categoría y, en general, la mayoría de ellos más agradables que los establecimientos americanos. La compañía extrajo, de una encuesta realizada a sus consumidores chinos, una lista con las veinte principales razones por las que visitaban sus establecimientos y obtuvo que el consumo de café ocupaba la sexta posición. La razón principal era el tener un lugar para reunirse. Alrededor del 90\% de las órdenes se consumen en los locales, frente al 20\% en Estados Unidos.

Una vez captados es cuando la compañía intenta educar a sus clientes en la cultura del café. La carta de bebidas se centra en las mismas bebidas basadas en café que en Estados Unidos, relegando al té a un segundo plano. La mayoría de los establecimientos de Starbucks en China continental, Taiwan y Hong Kong ofrecen un menú más amplio de comida y más espacio que sus equivalentes americanos.

El precio es uno de los primeros elementos que las compañías occidentales suelen adaptar. Sin embargo, Starbucks ha mantenido unos precios que pueden ser considerados excesivos para la población China. Un "grande latte" cuesta alrededor de 3.50\$ en Shangai donde el salario medio anual es de 3.800\$. Esto no parece ser un problema para uno de los mercados objetivo de Starbucks, la generación de pequeños emperadores, nacida a partir de las leyes chinas que permiten un único hijo y cuyos padres y abuelos han mimado en exceso. Los valores de individualismo que posee esta generación de jóvenes coinciden con los servicios y bebidas personalizados que ofrece Starbucks.

A pesar del éxito que tiene Starbucks en China, su corta historia dentro del país no ha estado libre de problemas debidos a las diferencias culturales. Podemos destacar la presión social que recibió tras haber ubicado uno de sus locales en el recinto de la Ciudad Prohibida en Pekín, que culminó con su cierre en 2007. Este establecimiento fue abierto durante el año 2000 dentro del recinto, cerca del Palacio de la Armonía Suprema, hecho que provocó la controversia y el rechazo de los ciudadanos a su presencia desde el primer momento.

A pesar de todo, el establecimiento continuó sus operaciones y fue en el año 2005 cuando decidieron retirar el logotipo y nombre del establecimiento con el objetivo de contentar a aquéllos que les acusaban de impacto negativo sobre el entorno del recinto histórico. La presión popular no disminuyó con esta medida. 
Finalmente, la decisión de cierre fue tomada a raíz de los comentarios aparecidos en el blog personal de Rui Chenggang, uno de los periodistas del canal estatal de noticias CCTV Channel 9. Chenggang señalaba en una entrada de su blog que la presencia de Starbucks en el recinto histórico era un "insulto para la civilización china”. Desde su publicación, el blog recibió más de quinientas mil visitas y una cantidad ingente de comentarios en menos de una semana.

La imagen de la compañía resultó dañada por la aparición en medios de comunicación locales e internacionales del descontento de los ciudadanos chinos. La principal conclusión a que podemos llegar es la persistencia de Starbucks en mantener las operaciones de su establecimiento en la Ciudad Prohibida durante más de siete años, a pesar de que, desde el principio, había importantes sectores de la ciudadanía china que tenían una opinión contraria.

Por último, merece la pena señalar que las comunicaciones se realizaron siempre mediante los responsables locales de la compañía, no emitiéndose comunicados haciendo referencia a este conflicto directamente desde la sede en Estados Unidos.

\subsection{Facebook en China}

Con cerca de 300 millones de usuarios, la red social Facebook se mantiene como el fenómeno de moda en Internet, pero no termina de triunfar en China, donde se encuentra la mayor comunidad de internautas del mundo, 360 millones de usuarios aproximadamente.

Sólo uno de cada mil internautas chinos utiliza Facebook, confirmando que sigue siendo un mundo virtual aparte, pese a los esfuerzos de las grandes empresas de la Web 2.0 por entrar en él. Ya ocurrió antes con el buscador Google que se ve superado en China por su rival local, Baidus. Baidu es el primer buscador de China y lidera el mercado de la publicidad online en el país asiático, con alrededor de dos tercios de los usuarios. Google Inc., líder en el mercado global, es el número dos en China, con una diferencia importante respecto a Baidu. Asimismo, Yahoo! Inc. se encuentra con la competencia de Sina y Sohu.

Las razones del fracaso de Facebook en China son similares a las apuntadas anteriormente: factores culturales y políticos, que abren, una vez más, una barrera entre los usuarios de Internet chinos y los de Occidente. En esencia, podría decirse que los jóvenes chinos prefieren el formato BBS (Bulletin Board System) y no tienen tanto interés en ampliar su círculo propio de amigos, una de las motivaciones de muchos usuarios de Facebook en otros países.

Además, los internautas chinos son consumidores -y no tanto creadores- de contenido y el fenómeno de la censura ayuda a mantener estas diferencias: en los foros, los chinos suelen hacer comentarios bajo seudónimos, mientras que en Facebook suele primar el usuario que hace público su nombre real y sus datos personales.

Hay ocasiones en que la censura ha afectado de forma más directa a Facebook: cuando lanzó su versión en chino mandarín, en el verano de 2008, fue bloqueada cuando tan sólo habían transcurrido unas horas. En cuestión de días el servicio regresó a la normalidad,

${ }^{5}$ En China, el Gobierno ejerce una amplia influencia sobre el conjunto de la sociedad y de la economía (Fanjul, 2002). 
pero el desafortunado debut en China pudo influir en que muchos usuarios se interesaran por sus competidores, ya que no se puede obviar que existen diferentes redes sociales en el país asiático (Xianoei.com, Chinay.com, FaceRen.com, Yeejee, etc.) que suponen una dura competencia debido a las trabas legales de China para que compañías extranjeras adquieran acciones de sus firmas locales. Así, merece la pena destacar las siguientes redes sociales:

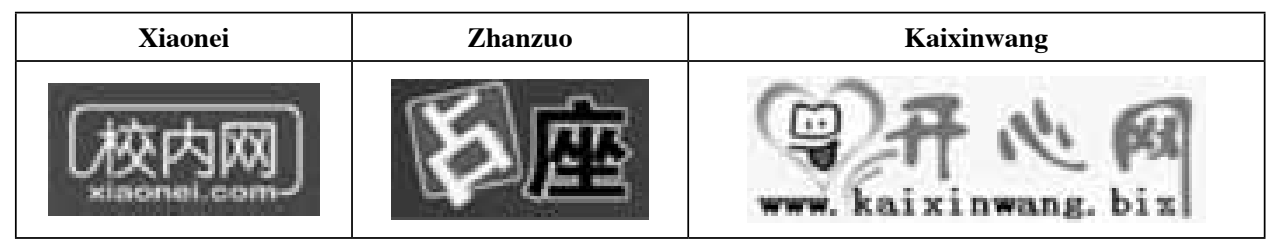

Antes de que Facebook fuera popular en el mundo, ya había redes sociales chinas tales como Xiaonei, cuyo origen data de diciembre de 2005 en la Universidad de Tsinghua (en Pekín) y que cuenta hoy día con unos quince millones de usuarios versus los trescientos mil que Facebook tiene en el país. Zhanzuo, tiene siete millones de miembros, y también son muy populares las páginas Web sociales Kaixinwang ("Red Feliz"). Algo similar ocurre con el negocio electrónico, donde empresas locales como Alibaba.com y Taobao.com tienen un notable éxito en China, lo que supone una barrera de entrada para empresas extranjeras.

Ventajas legales para las empresas chinas sobre las extranjeras y reticencias de los usuarios chinos para entrar en una red social internacional son algunas de las razones que dificultan que existan más internautas chinos en Facebook.

\section{CONCLUSIONES}

El tamaño real del mercado chino para productos occidentales es de trescientos millones de personas en la actualidad y no de 1.350 millones de personas y se prevé que la clase media-alta china llegue a ser de seiscientos millones de personas el año 2020. Se trata de una excelente masa crítica que está, a su vez, concentrada en grandes ciudades como Beijing, Shanghai, Guangzhou, Hong Kong y Chongqing, entre otras.

Las empresas deberían ser capaces de operar en un entorno global respetando la diversidad cultural, reconociendo que las necesidades personales, valores, estándares y expectativas están culturalmente condicionadas. Ello supone estar alerta al impacto del shock cultural en la gestión exitosa de las transiciones, desarrollar las habilidades necesarias y evitar el etnocentrismo.

La importancia de la elección de un buen nombre, tener en cuenta las particularidades que presenta el consumidor chino y no obviar el fenómeno de la censura y las ventajas legales de las empresas locales chinas versus las extranjeras resultan ser elementos cruciales para que las empresas occidentales logren entrar en el mercado chino con éxito.

La familia, la formación de grupos afines y las relaciones personales (guanxi), también son primordiales en la cultura china. De esta manera, el componente tradicional es un elemento común y consistente en los patrones de consumo y se convierte en un factor esencial. 
Además, se ha mostrado la importancia de establecer joint ventures con un socio local, así como la importancia de considerar los valores éticos y religiosos así como del tipo de poder político a través de cuatro casos de estudio.

Resumiendo, los retos de futuro de las empresas que deciden comercializar y vender sus productos en el gigante asiático pasan por tener en cuenta sus elementos culturales, por tratar de superar la barrera del idioma y por el establecimiento de relaciones con los socios locales (guanxi), entre otras.

\section{REFERENCIAS BIBLIOGRÁFICAS}

ADAMS, J. (2007): "Investing in Opportunity", Families in Business, Enero/Febrero, pp. 47-48.

BHASIN, B.B. (2007): "Succeeding in China: Cultural Adjustments for Indian Businesses", Cross Cultural Management: An International Journal, 14 (1), pp. 43-53.

BUENO CAMPOS, E.J.; SALMADOR SÁNCHEZ, M.P. Y GOLZMAN, A.A. (2006): "El Caso de las Empresas Españolas del Sector de la Alimentación en China”, Economía Industrial, 362 (Ejemplar dedicado a: China e India: Oportunidades y Estrategias), pp. 147-152.

BUSTELO, P. (2006): "Asignatura pendiente: la presencia económica de España en China e India", en: La presencia española en países de fuerte crecimiento: China e India, BUSTELO, P., CACHO, L. y ZABALLA, J.J. (eds.), Círculo de Empresarios, Madrid.

BUSTELO, P. (2008): "España ante el Auge Económico de China e India”, Boletín Económico de ICE, 2937, pp. 103-115.

COSMEN, A. (2002): "Perspectivas para la Empresa Española en China: la Experiencia de ALSA", Información Comercial Española: Revista de Economía, 797 (Ejemplar dedicado a: China en el siglo XXI), pp. 71-76.

CZINKOTA, M.R. Y RONKAINEN, I. A. (2002): Marketing Internacional, Prentice Hall, México, Sexta Edición.

DATAINVEX: http://datainvex.comercio.es fecha última consulta: 3 septiembre 2009.

DUNNING, J. H. (1988): "The Eclectic Paradigm of International Production: A Restatement of Some Possible Extensions", Journal of International Business Studies, 19, Spring, pp. 1-31.

ERNST \& YOUNG (2008): "European Attractiveness Survey 2008”, Ernst \& Young. Disponible online en: http://www.ey.com.

ERNST \& YOUNG (2009): "European Attractiveness Survey 2009”, Ernst \& Young. Disponible online en: http://www.ey.com.

FANJUL MARTÍN, E. (2002): “Algunas Cuestiones Clave para Abordar el Mercado Chino", Información Comercial Española: Revista de economía, 797 (Ejemplar dedicado a: China en el siglo XXI), pp. 55-62.

FANJUL, E. (2009): "El Consenso de Pekín: ¿Un Nuevo Modelo para los Países en Desarrollo?, ARI 122/2009, Cooperación Internacional y Desarrollo/Asia-Pacífico, Real Instituto Elcano.

GRANDE, I. (2004): Marketing Croscultural, Libros Profesionales de Empresa, ESIC Editorial.

HARRIS, P. Y MORAN, R. (2001): “European Leadership in Globalization”, en: Albrecht, M.H. (Ed). International HRM. Managing diversity in the Workplace, Blackwell Publishers Ltd. pp. 41-54.

HERNÁNDEZ-NIETO, J. O. (2006): "Riesgos y Consideraciones sobre la Estrategia de Entrada de Empresas Españolas en China”, Economía Industrial, 362 (Ejemplar dedicado a: China e India: Oportunidades y Estrategias), pp.103-109.

JOHNSON, J. Y TELLIS, G.J. (2008): "Drivers of Success for Market Entry into China and India“, Journal of Marketing: A Quarterly Publication of the American Marketing Association, 72 (3), pp. 1-13. 
KPMG (2008): "Global Corporate Capital Flows, 2008/9 to 2013/14. A Study of the Investment Intentions of Companies in 15 Countries around the World", KPMG. Disponible online en: http://www.kpmg.com.

LA CAIXA (2006): "El Dragón Chino Asoma su Cabeza en España", Informe Mensual de La Caixa, Servicio de Estudios.

ORDOÑEZ DE PABLOS, P. (2004): "La Importancia de Guanxi, Renqing y Xinyong en las Relaciones Empresariales en China: Implicaciones para las Empresas Españolas". Información Comercial Española: Revista de Economía, 818, pp. 221-234.

PRICEWATERHOUSECOOPERS (2008): "11th Annual Global CEO Survey, PriceWaterhouseCoopers". Disponible online en: http://www.pwc.com/gx/en/ceo-survey/pdfs/11th ceo survey. pdf

PRICEWATERHOUSECOOPERS (2009): "12th Annual Global CEO Survey, PriceWaterhouseCoopers". Disponible online en: http://www.pwc.com/gx/en/ceo-survey/pdfs/doca.pdf

QUER, D.; CLAVER, E. Y RIENDA, L. (2007a): "Business and Management in China: A Review of Empirical Research in Leading International Journals", Asia Pacific Journal of Management, 24 (3), pp. 359-384.

QUER RAMÓN, D.; CLAVER CORTÉS, E. Y RIENDA GARCÍA, L. (2007b): "Multinacionales Chinas: El Nuevo Reto del Escenario Global”, Boletín económico de ICE, Información Comercial Española, 2925, pp. 11-24.

QUER RAMÓN, D.; CLAVER CORTÉS, E. Y RIENDA GARCÍA, L. (2009): "Negocios y Dirección Estratégica de Empresas en China e India", Boletín económico de ICE, Información Comercial Española, 2957 (Ejemplar dedicado a: La Respuesta Europea a la Crisis Financiera y Económica. El Sistema Económico Internacional en 2008), pp. 39-54.

ROOT, F.R. Y AHMED (1979): "Empirical Determinants of Manufacturing Direct Foreign Investment in Developing Countries", Economic Development and Cultural Change, 27, (July), 75154.

SÁNCHEZ-MONASTERIO, M. Y CASABURI, I. (2008a): "Retos de la Dirección de Marketing en China (1 $1^{a}$ parte)", Harvard Deusto Marketing y Ventas, 84, pp. 62-68.

SÁNCHEZ-MONASTERIO, M. Y CASABURI, I (2008b): "Retos de la Dirección de Marketing en China (2a parte)", Harvard Deusto Marketing \& Ventas, 85, pp. 12-19.

SÁNCHEZ-MONASTERIO, M. (2009a): "Claves de la Dirección de Marketing en China (1 $1^{\text {a }}$ parte)", Global Asia.

SÁNCHEZ-MONASTERIO, M. (2009b): "Claves de la Dirección de Marketing en China (2 ${ }^{\mathrm{a}}$ parte)", Global Asia.

SÁNCHEZ-MONASTERIO, M. (2009c): “Claves de la Dirección de Marketing en China (3 parte)”, Global Asia.

THE WORLD FACTBOOK (2009): http://www.odci.gov/cia/publications/factbook. Washington, D.C. Central de Inteligencia Americana. Fecha última consulta: 1 de septiembre de 2009.

ZHAO, H.; YADONG, L. Y SUH, T. (2004): "Transaction Cost Determinants and Ownership-Based Entry Mode Choice: A Meta-Analytical Review”, Journal of International Business Studies, 35 (6), November, pp. 524-544. 